MDPI

MOL2NET, International Conference Series on Multidisciplinary Sciences

\title{
sciforum
}

\section{A new procedure for the Antioxidant capacity dpph assessment in small samples}

\author{
Gombert $M^{a}$., Carrasco-Garcia $A^{b}$., Carrasco-Luna Ja,c. Codoñer-Franch $\mathrm{P}^{\mathrm{a}, \mathrm{b}}$.
}

a. Dpto Pediatria Obstetricia y Ginecologia. Facc. Medicina y Odontologia. Universitat de Valencia

b. FISABIO. Fundació per al foment de la Investigació Sanitaría I biomedical de la Comunitat Valenciana.

c. Dpto Ciencias Experimentales. Facc de Ciencias Experimentales y Veterinaria UCV.

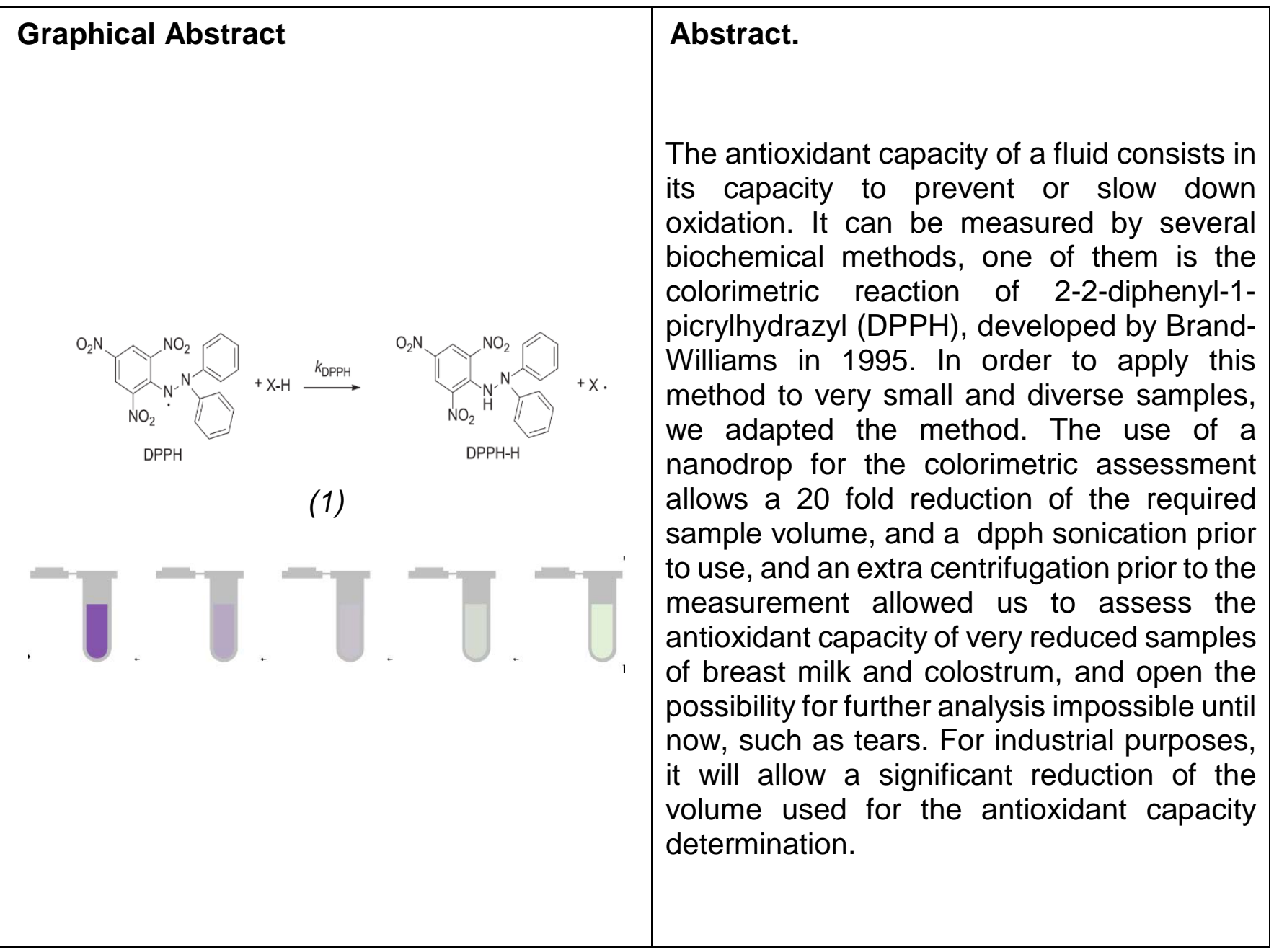

Introduction (optional)

The antioxidant capacity of a substance is its ability to prevent or slow down oxidation. It is a reaction of a transfer of electrons. The molecule catching electrons is referred to as the oxidative agent and the molecule giving electrons as the reducing agent. Oxidative stress is toxic for the living cells, and it can be limited by different pathways: the inhibition of enzymes responsible of the production of oxidative species, the synthesis, activation or stabilization of antioxidant enzymes, and finally by a direct scavenging of radical species.(1) 
In living organisms, especially in association to the respiration process, free radicals are commonly produced. Antioxidants are naturally present to prevent damages, and therefore the antioxidant capacity of a substance is a key element to evaluate. Several methods can be employed to assess total antioxidant capacity in a liquid, one of the most reliable and frequently used is based on a colorimetric reaction involving 2-2-diphenyl-1-picrylhydrazyl (DPPH) (2).

Brand \& Williams developed in 1995 (3) the method of assessment of antioxidant capacity based on the color change of DPPH according to its oxidation status. The oxidized DPPH is a radical, with dark purple color and in contact with antioxidant species, reduced in contact with antioxidant species, turning to yellow-green color. The amount of oxidized DPPH can be quantified at 517nm spectrophotometrically. Using a known dose of antioxidant molecule, such as Trolox, a standard curve of antioxidant can be obtained. The antioxidant capacity of any sample can be assessed measuring the oxidized DPPH, and the result is expressed in Trolox equivalents. This method has been adapted by Martinez et al. in 2005 in order to assess antioxidant capacity in plasma samples (4).

Despite this modification, we encountered difficulties to proceed to this measurement on small samples of breast milk. The obstacles we have met using this method are (i) the important volume of sample required and (ii) the interference of molecules of fat present in some biological fluids (breast milk). We therefore adapted the original method in order to perform antioxidant capacity assessment on our samples. Here is presented the adapted protocol, and an example of results obtained.

Materials and Methods (optional)

\begin{tabular}{|c|c|c|}
\hline \multicolumn{3}{|c|}{ Table 1.Trolox standard curve } \\
\hline Trolox $(\mu \mathrm{M})$ & Methanol $(\mu \mathrm{L})$ & $\begin{array}{c}\text { Trolox mother } \\
\text { solution }(\mu \mathrm{M})\end{array}$ \\
\hline 800 & 0 & 1000 \\
\hline 700 & 125 & 875 \\
\hline 600 & 250 & 750 \\
\hline 500 & 375 & 625 \\
\hline 400 & 500 & 500 \\
\hline 300 & 625 & 375 \\
\hline 200 & 750 & 250 \\
\hline 100 & 875 & 125 \\
\hline 75 & 917 & 83 \\
\hline 50 & 937 & 63 \\
\hline 25 & 969 & 31 \\
\hline 10 & 987 & 13 \\
\hline 1 & 999 & 1 \\
\hline 0 & 1000 & 0 \\
\hline
\end{tabular}

Table 1: Standard curve concentrations of Trolox

Protocol

1. Label 1,5ml Eppendorf from 0 to $800 \mu \mathrm{M}$ as indicated in table 1 , and $0,5 \mathrm{ml}$ Eppendorf for the DPPH reaction (14 for the standard curve + the number of samples)

2. Prepare $200 \mathrm{ml}$ of $80 \%$ methanol 
3. dissolve $10 \mathrm{mg}$ Trolox in $50 \mathrm{ml}$ methanol $80 \%$

4. In each tube of the standard curve, add Trolox and methanol following the proportions indicated in table 1

5. Vortex pulse each tube

6. Dissolve $3,9 \mathrm{mg}$ dpph in $100 \mathrm{ml}$ methanol $80 \%$ and sonicate 10 minutes

7. Pipet $10 \mu \mathrm{L}$ of sample or standard $+290 \mu \mathrm{L}$ of dpph in the labelled $0,5 \mathrm{ml}$ ependorfs

8. Vortex pulse each tube

9. Incubate the standard curve and the samples $30 \mathrm{~min}$ at room temperature in the dark

10. Centrifuge the samples $10 \mathrm{~min}$ at 5000G in order to prevent artefacts due to the nature of the sample

11. Measure the absorbance with a nanodrop at 517 nanometer : $2,5 \mu l$ in the reader.

12. A curve is obtained from the standard Trolox solution absorbance, and the equation of this curve is used to obtain the value of antioxidant capacity of each sample, expressed in Trolox equivalent.

\section{Results and Discussion (optional)}

Measuring antioxidant capacity in breast milk using DPPH reaction presented two issues: An important volume of samples required for the test, and the artefacts produced by the complex composition of certain samples.

Reading about DPPH reaction and experiment, and performing different tests, we developed an adapted protocol which is adapted for small volumes of diverse body fluids.

To begin, fluid samples from living beings are complex, with lipids and proteins in suspension, susceptible to interfere with the colorimetric measurement. By performing a supplementary centrifugation at the end of the colorimetric reaction (5000G 10min), at a speed allowing separation of the components, a clean measurement is obtained.

Moreover, the $200 \mu \mathrm{l}$ volume required for the assessment in the previous method limits the number of experiment that can be done on one sample, and sometimes makes it impossible, especially in the colostrum samples. The use of the nanodrop device and the adaptation of the volume of reactants allows a determination with a sample volume of only $10 \mu \mathrm{l}$.

Finally, the sonication of the DPPH solution is not specified in most of the protocols, nevertheless we could observe that it is necessary to obtain an homogeneous solution, therefore we want to insist on the importance of this step to obtain reliable results.

Using the protocol described earlier, we determined antioxidant capacity in 10 breast milk samples (table 2). In order to assess the reliability of the determination in a diluted sample, we diluted all sample in a $1 / 2$ ratio with the solvent (methanol $80 \%$ ). The measurements were performed with a nanodrop (a) and with a classic spectrophotometer (b).

First, we observed that the measurement of the antioxidant capacity of a diluted sample does not permit to calculate the value of the original sample, we therefore recommend not to proceed to dilution. In the case of very small volume of sample, the experiment can be done with our method, as the determination with the Nanodrop allows an assessment of antioxidant capacity equivalent to the one done with a classic spectrophotometer.

Table 2. Antioxidant capacity assessment with two different espectrofotometer devices.

\begin{tabular}{|c|c|c|c|c|c|c|c|}
\hline \multirow[t]{3}{*}{$\mathbf{N}^{\circ}$} & \multicolumn{3}{|c|}{ Nanodrop determination (a) } & \multicolumn{4}{|c|}{ Classic spectrophotometer determination (b) } \\
\hline & Without dilution & \multicolumn{2}{|c|}{ diluted } & \multicolumn{2}{|c|}{ Without dilution } & \multicolumn{2}{|c|}{ diluted } \\
\hline & $\begin{array}{ccc}\text { abs(517) } & \begin{array}{c}\text { Trolox } \\
\text { equivalent }\end{array}\end{array}$ & $\operatorname{abs}(517)$ & $\begin{array}{c}\text { Trolox } \\
\text { equivalent }\end{array}$ & $\operatorname{abs}(517)$ & $\begin{array}{c}\text { Trolox } \\
\text { equivalent }\end{array}$ & $\operatorname{abs}(517)$ & $\begin{array}{c}\text { Trolox } \\
\text { equivalent }\end{array}$ \\
\hline
\end{tabular}




\begin{tabular}{|c|cc|cc|cc|cc|}
\hline $\mathbf{1}$ & 0,2 & 105,7 & 0,5 & 44,7 & 0,2 & 126,0 & 0,5 & 46,8 \\
$\mathbf{2}$ & 0,4 & 73,7 & 0,7 & 30,4 & 0,4 & 71,1 & 0,7 & 25,9 \\
$\mathbf{3}$ & 0,5 & 57,7 & 0,6 & 32,6 & 0,5 & 58,5 & 0,6 & 32,8 \\
$\mathbf{4}$ & 0,2 & 112,2 & 0,8 & 14,0 & 0,2 & 114,9 & 0,8 & 12,4 \\
$\mathbf{5}$ & 0,3 & 79,9 & 0,9 & 11,4 & 0,4 & 72,2 & 0,8 & 11,2 \\
$\mathbf{6}$ & 0,0 & 230,7 & 0,8 & 14,0 & 0,1 & 179,1 & 0,7 & 15,9 \\
$\mathbf{7}$ & 0,1 & 214,7 & 0,8 & 14,9 & 0,1 & 202,3 & 0,7 & 21,8 \\
$\mathbf{8}$ & 0,1 & 190,7 & 0,8 & 18,4 & 0,2 & 147,7 & 0,8 & 15,1 \\
$\mathbf{9}$ & 0,4 & 75,7 & 0,8 & 14,0 & 0,5 & 56,8 & 0,7 & 16,3 \\
$\mathbf{1 0}$ & 0,4 & 71,7 & 0,9 & 7,4 & 0,5 & 58,1 & 0,8 & 10,4 \\
\hline
\end{tabular}

Table 2: Antioxidant capacity of 10 breast milk samples. Determination with nanodrop (a) and classic spectrophotometer (b) of DPPH absorbance and Trolox equivalent.

Moreover, the present method also allows antioxidant capacity determination in other body fluids, as referenced in table 3 . The last centrifugation prior to measurement allows to limit the interferences due to the nature of the samples.

\begin{tabular}{|ccccc|}
\hline \multicolumn{5}{|c|}{ Table 3 Method application to body fluids } \\
\hline Sample & $\begin{array}{c}\text { Trolox } \\
\text { equivalents } \\
\text { average }\end{array}$ & std desv & Type error \% \\
Culture medium & 175,2 & \pm & 12,8 & 7.30 \\
Urine & 1120,2 & \pm & 59,4 & 5.30 \\
Breast milk & 457,6 & \pm & 33,1 & 7.23 \\
Serum & 405,9 & \pm & 29,6 & 7.29 \\
Saliva & 271,9 & \pm & 12,2 & 4.48 \\
\hline
\end{tabular}

The applicability to very diverse and small samples opens the potential application of DPPH based antioxidant capacity measurement with a type error between 4-8 \%. For instance, saliva of very young children, tears, colostrum, or other body fluids available only in reduced quantity could now be analyzed. The reduced volume required also allows to perform replicated, and also other tests. This advantage can also benefit the industry, with the assessment of the antioxidant capacity of dairy products, or of food additives (5) using reduced volumes.

\section{Conclusions (optional)}

We present an adaptation of the method of Brand-Williams (1995) based on the colorimetric assessment of the DPPH oxidative reaction in order to quantify the antioxidant capacity of a liquid. Our proposal consist on the use of a NanoDrop for colorimetric assessment, reducing 20 times the volume necessary for the test. Plus, we underline the interest of adding a centrifugation prior to the measurement, in order to prevent artefacts caused by the nature of several body fluids. 
1. Advantages and limitations of common testing methods for antioxidants R. Amorati \& L. Valgimigli. Free Radical Research, May 2015; 49(5): 633-649 @ 2015 Informa UK, Ltd. ISSN 1071-5762 print/ISSN 1029-2470 online DOI: 10.3109/10715762.2014.996146

2. METHODS FOR DETERMINATION OF ANTIOXIDANT CAPACITY: A REVIEW

Deepshikha Gupta, IJPSR, 2015; Vol. 6(2): 546-566. E-ISSN: 0975-8232; P-ISSN: 23205148

3. Brand-Williams W, Cuvelier ME and Berset C: Use of a free radical method to evaluate antioxidant activity. Food Science and Technology. 1995; 28: 25-30

4. S. Martinez, L. Valek, J. Resetic, D. Ferenec Ruzic. Cyclic voltammetry study of plasma antioxidant capacity - Comparison with the DPPH and TAS spectrophotometric methods. Joumal of Electroanalytical Chemistry 588 (2006) 68-73.

5. Shimamura T, Sumikura Y, Yamazaki T, Tada A, Kashiwagi T, Ishikawa $H$, Matsui $\mathrm{T}$, Sugimoto $\mathrm{N}$, Akiyama $\mathrm{H}$, Ukeda $\mathrm{H}$. Applicability of the DPPH assay for evaluating the antioxidant capacity of food additives - inter-laboratory evaluation study -. Anal Sci. 2014;30(7):717-21. 